\title{
Comparison of CdZnTe neutron detector models using MCNP6 and Geant4
}

\author{
Emma Wilson*, Mike Anderson* ${ }^{*}$, David Prendergast ${ }^{\dagger}$ and David Cheneler* \\ * Department of Engineering, Lancaster University, Lancaster, LA1 4YW, UK. E-mail: e.wilson6@lancaster.ac.uk \\ ${ }^{\dagger}$ Innovative Physics Ltd., Shanklin, Isle of Wight, PO37 7JB, UK.
}

\begin{abstract}
The production of accurate detector models is of high importance in the development and use of detectors. Initially, MCNP and Geant were developed to specialise in neutral particle models and accelerator models, respectively; there is now a greater overlap of the capabilities of both, and it is therefore useful to produce comparative models to evaluate detector characteristics. In a collaboration between Lancaster University, UK, and Innovative Physics Ltd., UK, models have been developed in both MCNP6 and Geant4 of Cadmium Zinc Telluride (CdZnTe) detectors developed by Innovative Physics Ltd. Herein, a comparison is made of the relative strengths of MCNP6 and Geant4 for modelling neutron flux and secondary $\gamma$-ray emission. Given the increasing overlap of the modelling capabilities of MCNP6 and Geant4, it is worthwhile to comment on differences in results for simulations which have similarities in terms of geometries and source configurations.
\end{abstract}

\section{INTRODUCTION}

In order to maintain and improve safety standards within the nuclear sector, more emphasis is placed on novel detector materials and configurations for use in nuclear power, decommissioning, and security, as well as for other applications. It is for this reason that the capability to accurately mathematically model detector response is essential; in the first instance this would be a check of the applicability of the detector for the planned purpose.

Both MCNP6 [1] and Geant4 [2] are widely used nuclear and radiation simulation tools, and both have their specialisations which arise from the background and purpose of their development. An understanding of the difference capabilities and, where appropriate, direct comparisons of these tools is essential, as it give an indication of which is more appropriate in a given circumstance. Previous work in this field has compared the capabilities of MCNP and Geant4 in areas including medicine [3], nuclear power [4], and security [5], although the development of both MCNP and Geant4, and the associated cross-section data, is on-going such that the individual capabilities of the codes vary.

Cadmium zinc telluride $(\mathrm{CdZnTe}$, or $\mathrm{CZT})$ radiation detectors are widely used in $\gamma$-ray spectroscopy as a roomtemperature, semiconductor-based detector. This is of particular use as $\mathrm{CdZnTe}$ detectors possess an improved energy resolution, when compared to scintillation-based radiation detectors, and, unlike high-purity germanium, has a sufficiently wide band-gap to significantly reduce thermal excitation of electrons across the gap, and therefore CdZnTe detectors do
TABLE I

THE HIGHEST INTENSITY PROMPT SECONDARY $\gamma$-RAYS PRODUCED AS A RESULT OF NEUTRON CAPTURE ON A CDZNTE DETECTOR.

\begin{tabular}{|c||c||c||c|}
\hline Reaction & $\mathrm{E}_{\gamma}(k e V)$ & Reaction & $\mathrm{E}_{\gamma}(\mathrm{keV})$ \\
\hline${ }^{112} \mathrm{Cd}(\mathrm{n}, \mathrm{n} \gamma)$ & 617.5 & ${ }^{110} \mathrm{Cd}(\mathrm{n}, \mathrm{n} \gamma)$ & 657.8 \\
${ }^{114} \mathrm{Cd}(\mathrm{n}, \mathrm{n} \gamma)$ & 558.5 & ${ }^{111} \mathrm{Cd}(\mathrm{n}, \mathrm{n} \gamma)$ & 245.4 \\
${ }^{128} \mathrm{Te}(\mathrm{n}, \mathrm{n} \gamma)$ & 743.2 & ${ }^{126} \mathrm{Te}(\mathrm{n}, \mathrm{n} \gamma)$ & 666.3 \\
${ }^{130} \mathrm{Te}(\mathrm{n}, \mathrm{n} \gamma)$ & 839.4 & ${ }^{64} \mathrm{Zn}(\mathrm{n}, \mathrm{n} \gamma)$ & 991.6 \\
${ }^{112} \mathrm{Cd}(\mathrm{n}, \mathrm{n} \gamma)$ & 606.9 & ${ }^{113} \mathrm{Cd}(\mathrm{n}, \mathrm{n} \gamma)$ & 558.3 \\
${ }^{114} \mathrm{Cd}(\mathrm{n}, \mathrm{n} \gamma)$ & 576.1 & ${ }^{113} \mathrm{Cd}(\mathrm{n}, \mathrm{n} \gamma)$ & 651.2 \\
${ }^{128} \mathrm{Te}(\mathrm{n}, \mathrm{n} \gamma)$ & 753.8 & ${ }^{111} \mathrm{Cd}(\mathrm{n}, \mathrm{n} \gamma)$ & 245.3 \\
${ }^{130} \mathrm{Te}(\mathrm{n}, \mathrm{n} \gamma)$ & 748.8 & & \\
\hline
\end{tabular}

not require liquid nitrogen cooling [6].The mechanism for the detection of neutrons with $\mathrm{CdZnTe}$ detectors is via thermal neutron capture on ${ }^{113} \mathrm{Cd}$, resulting in a series of $\gamma$-rays which can then be detected in the same manner as with standard $\gamma$ ray spectroscopy. CdZnTe neutron detection has been used in a variety of areas, including around medical accelerators [7] and for the detection of special nuclear materials [8].

\section{Materials And Methodology}

Both models use a ${ }^{252} \mathrm{Cf}$ neutron source and a $\mathrm{CdZnTe}$ detector, in this instance composed of $48 \% \mathrm{Cd}, 2 \% \mathrm{Zn}$, and the remainder comprised of Te. Of particular interest is the production of secondary particles within the detector.

The mechanism by which $\mathrm{CdZnTe}$ detects neutrons is via neutron capture and the emission of secondary $\gamma$-rays from the resulting excited nucleus, which will then result in the production of photoelectrons [9], [10]. ${ }^{113} \mathrm{Cd}$ has an exceptionally high neutron capture cross-section of 20615 barns at thermal neutron energies [11] [12] and such an event results in characteristic $\gamma$-rays of the following energies [13]:

$$
\begin{aligned}
{ }^{113} C d+ & n \rightarrow{ }^{114} C d^{*} \\
& \rightarrow \gamma_{1}(558.6 \mathrm{keV})+\gamma_{2}(651.3 \mathrm{keV})+\text { further } \gamma
\end{aligned}
$$

Further characteristic $\gamma$-rays emitted from CdZnTe from thermal neutron capture are shown in Table I [7], [13], [14]. Most of these $\gamma$-ray transitions decay through the first excited state in ${ }^{114} \mathrm{Cd}$.

The proportions of the constituent materials which make a CdZnTe is important, as this makes a difference to detection efficiency [15]. 


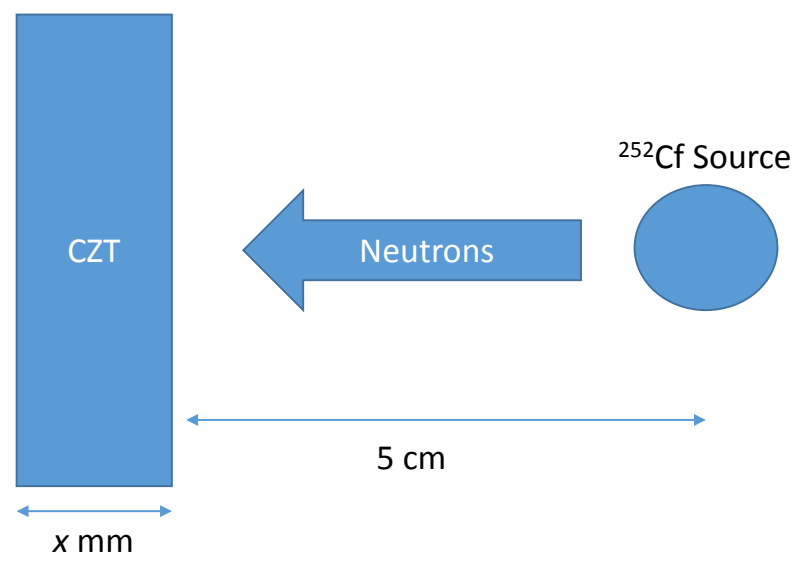

Fig. 1. A simplified sketch of the set-up used in both MCNP and Geant4 simuations.

Figure 1 shows a basic version of the setup used. The neutron source emits either a range of energies from a ${ }^{252} \mathrm{Cf}$ source, which are describes in reference [16]. The ${ }^{252} \mathrm{Cf}$ Lancaster University neutron source may be used in order to best compare theoretical models with experimental results. The thickness of a CdZnTe detector varies, but the value of $1 \mathrm{~mm}$ is within the thickness range commonly used [17], [18], [19].

\section{A. $M C N P$}

MCNP6 is a combination of the capabilities of MCNP5 and MCNPX, as well as the further development of both of these codes by groups at Los Alamos National Laboratory, where neutron transport codes have been developed since the 1950s [20]. Later development of photon transport codes, and the merger of the two lead to the release of the MCNP3 (originally Monte Carlo Neutron Photon, now Monte Carlo N-Particle) in 1983.

Figure 2 shows cross-section comparisons of the ENDF/BVII.1 and JENDL-3.3 cross section files. The cross-sections used in MCNP6 are selected while building the input file, with the cross-section data being selected as the isotopes, or ZAID, of each material are built.

The neutron discrete energy cross section libraries for most nuclei are from the ENDF/B-VII.0 or ENDF/B-VII.1, the latter of which is the most recent. There are various cross-section libraries for reactions taking place at different temperatures [23], [24]. In these simulations, the cross-sections used for each material are listed in table II.

\section{B. Geant4}

Geant4 is a Monte Carlo simulation toolkit for the simulation of particles travelling through and interacting with matter. It is a $\mathrm{C}++$ implemented open-source code, developed by a worldwide collaboration of physicists. Geant 4 is used within a wide variety of scientific fields, in topics as diverse as high-energy particle physics, nuclear physics, medical science,

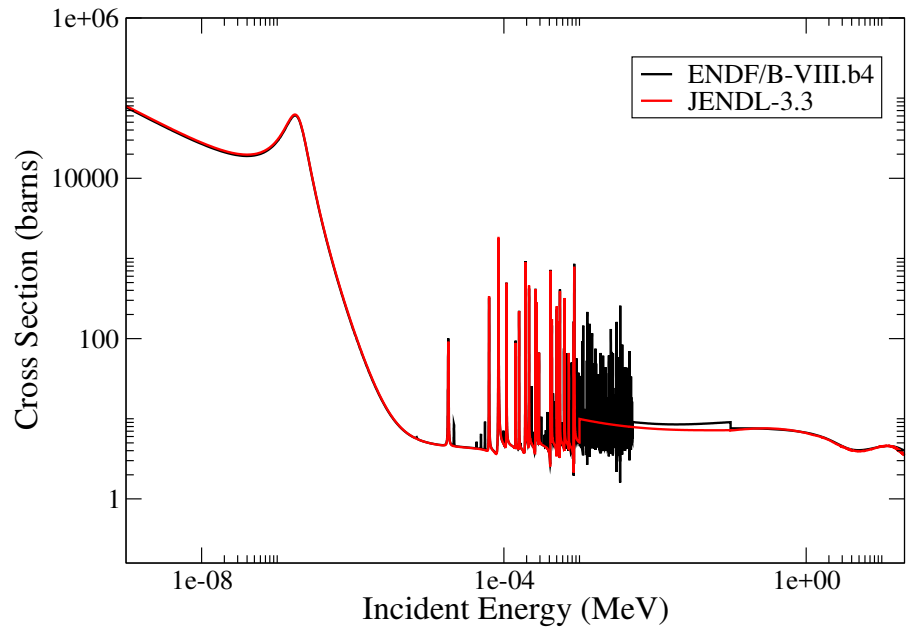

Fig. 2. The cross-section libraries ENDF/B-VII.1 [23] and JENDL-3.3 [25] available for the ${ }^{114} \mathrm{Cd}(\mathrm{n}$,total) reaction. Data extracted from International Atomic Energy Authority database [21].

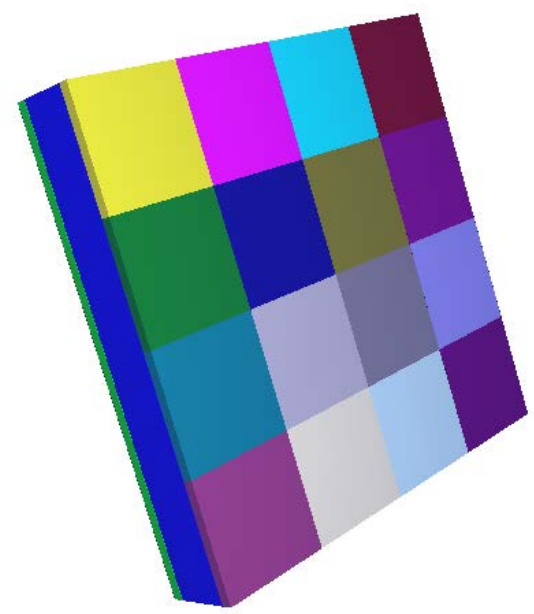

Fig. 3. A simple model produced in MCNP6, where the detector is in the centre, and on either side are pixellated slices of air, the number of which varied, used to produce the radiation flux on the front and rear of the detector.

TABLE II

THE MCNP6 MATERIALS USED TO MODEL THE DETECTOR, AND THEIR RESPECTIVE CROSS-SECTION INFORMATION. ALSO INCLUDING THE RATIO OF EACH MATERIAL USED IN THESE SIMULATIONS.

\begin{tabular}{|c||c||c||c|}
\hline Material & Cross-section source & Temperature (K) & Ratio (\%) \\
\hline $\mathrm{Zn}$ & ENDF/B-VII.0 & 293.6 & 5 \\
$\mathrm{Cd}$ & ENDF/B-V & 300 & 45 \\
$\mathrm{Te}$ & ENDF/B-VII.1 & 293.6 & 50 \\
\hline
\end{tabular}


$1 \mathrm{~mm} \mathrm{CdZnTe}$

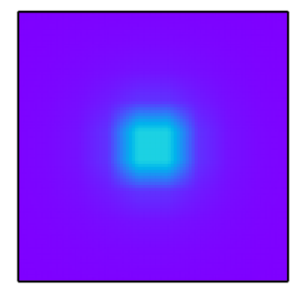

$5 \mathrm{~mm} \mathrm{CdZnTe}$

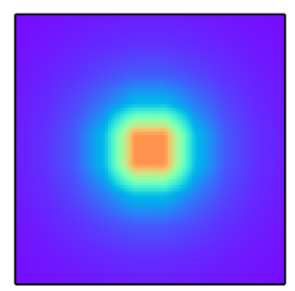

$10 \mathrm{~mm} \mathrm{CdZnTe}$

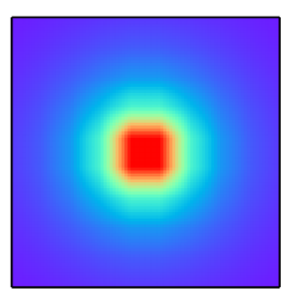

Fig. 4. The simulation of neutron captures using MCNP6, in various thickness of CdZnTe detector. For the purpose of this simulation, the detector was split into $10 \times 10$ pixels.

and accelerator physics. The version used in the simulations described here is Geant4-10.3, released December 2016, patch 1, released February 2017.

Geant4 allows for the modelling of secondary particles. Various physics lists are available for elastic and inelastic hadron scattering; for the relatively low-energy neutrons used here the BERT (Bertini cascade) and BIC (Binary cascade) models are applicable. These both allow for the modelling of protons, neutrons and pions, with BERT also being able to model kaons and hyperons. The energy ranges are $0-15 \mathrm{GeV}$ for BERT, and 0 - $5 \mathrm{GeV}$ for BIC [26]. The PhysicsList() used in this instance uses a combination of high precision neutron physics (HP), for the neutron interactions, and electromagnetic physics, for the electrons, and $\gamma$-rays within the simulation.

The cross-section data for the simulation comes from a variety of sources, depending on the interaction of interest. For the neutron interactions, this comes from G4NDL4.5, which is mainly based on the ENSDF/B-VII library. Also of use is the TENDL1.3 cross-section data set, which is used in Geant4 for the cross-sections of incident particles. TENDL1.5 crosssections for neutron capture, and subsequent $\gamma$-ray emission, on ${ }^{113} \mathrm{Cd}$ are illustrated in figure 2 .

\section{RESUlts AND ANALYSIS}

\section{A. $M C N P$}

Figure 4 shows the capture of neutrons from a ${ }^{252} \mathrm{Cf}$ neutron source in various thickness of $\mathrm{CdZnTe}$ detector material. This is the results of the simulation of $10^{10}$ iniital neutrons. The expectation would be that the thicker a shield or detector is, the more neutron capture events occur. The simulation agrees with this expectation.

Figure 5 shows the fluxes of neutrons from ${ }^{252} \mathrm{Cf}$ source in various thickness of CdZnTe. This is the results of the
$1 \mathrm{~mm} \mathrm{CdZnTe}$

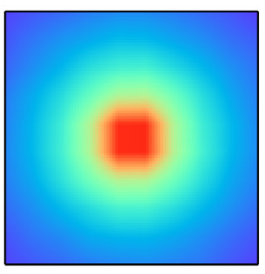

$5 \mathrm{~mm} \mathrm{CdZnTe}$

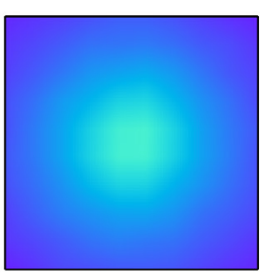

$2 \mathrm{~mm}$ CdZnTe

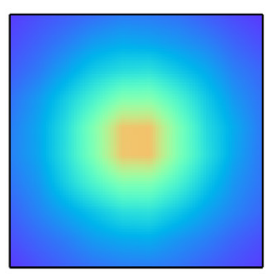

$10 \mathrm{~mm} \mathrm{CdZnTe}$

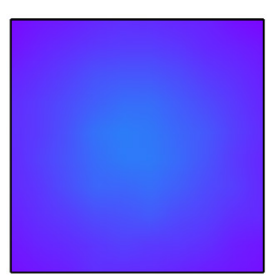

25

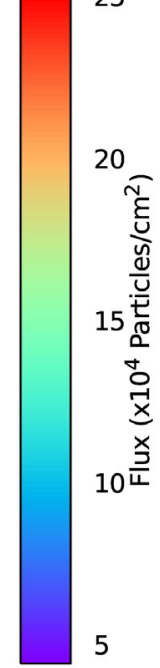

Fig. 5. The simulation of neutron fluxes using MCNP6, on the rear of $1 \mathrm{~mm}$, $2 \mathrm{~mm}, 5 \mathrm{~mm}$, and $10 \mathrm{~mm}$ CZT blocks. For the purpose of this simulation, the region at the rear of the detector was split into $10 \times 10$ pixels.

TABLE III

DETAILING THE MAJOR PEAKS OBSERVED IN THE $\gamma$-RAY SPECTRUM PRODUCED AS A RESULT OF THE NEUTRON CAPTURE ON A CDZNTE DETECTOR. PEAK FITTING OF THE THEORETICAL RESULTS WAS PERFORMED USING ROOT, WITH THE ENERGIES AND THE ERROR MEASUREMENTS ON THE ENERGIES COMING FROM THIS PEAK FITTING.

\begin{tabular}{|c||c||c|}
\hline $\mathrm{E}_{\gamma}(k e V)$ & Reaction & Experimental Energy (keV) \\
\hline $245.473(4)$ & ${ }^{111} \mathrm{Cd}(\mathrm{n}, \mathrm{n} \gamma)$ & $245.395(20)[27]$ \\
$558.334(3)$ & ${ }^{114} \mathrm{Cd}(\mathrm{n}, \mathrm{n} \gamma)$ & $558.456(2)[13]$ \\
$606.931(1)$ & ${ }^{112} \mathrm{Cd}(\mathrm{n}, \mathrm{n} \gamma)$ & $606.821(6)[29]$ \\
$617.651(3)$ & ${ }^{112} \mathrm{Cd}(\mathrm{n}, \mathrm{n} \gamma)$ & $617.518(3)[29]$ \\
$650.978(1)$ & ${ }^{114} \mathrm{Cd}(\mathrm{n}, \mathrm{n} \gamma)$ & $651.256(5)[13]$ \\
$657.607(8)$ & ${ }^{110} \mathrm{Cd}(\mathrm{n}, \mathrm{n} \gamma)$ & $657.7600(11)[30]$ \\
$666.255(30)$ & ${ }^{126} \mathrm{Te}(\mathrm{n}, \mathrm{n} \gamma)$ & $666.331(12)[31]$ \\
$742.966(8)$ & ${ }^{128} \mathrm{Te}(\mathrm{n}, \mathrm{n} \gamma)$ & $743.22(2)[32]$ \\
$748.968(8)$ & ${ }^{130} \mathrm{Te}(\mathrm{n}, \mathrm{n} \gamma)$ & $748.76(2)[33]$ \\
$753.654(9)$ & ${ }^{128} \mathrm{Te}(\mathrm{n}, \mathrm{n} \gamma)$ & $753.82(2)[32]$ \\
$839.429(1)$ & ${ }^{130} \mathrm{Te}(\mathrm{n}, \mathrm{n} \gamma)$ & $839.49(2)[33]$ \\
$991.853(16)$ & ${ }^{64} \mathrm{Zn}(\mathrm{n}, \mathrm{n} \gamma)$ & $991.56(5)[34]$ \\
\hline
\end{tabular}

simulation of $10^{10}$ iniital neutrons. As would be expected, a greater neutron flux is observed on the rear of the detector when the detector thickness is smaller.

\section{B. Geant4}

Figure 6 shows the $\gamma$-spectrum resulting from the interaction of neutrons from a ${ }^{252} \mathrm{Cf}$ source and a CdZnTe detector. The details of the peaks in the spectrum are described in table III; the $\gamma$-rays resulting from the de-excitation of the most common cadmium, zinc, and tellurium have been wellcharacterised, as described in the relevent Nuclear Data Sheets [13], [27], [29], [30], [31], [32], [33], [34], and the results presented here are in agreement with the energies of the most prominent of these $\gamma$-rays. 


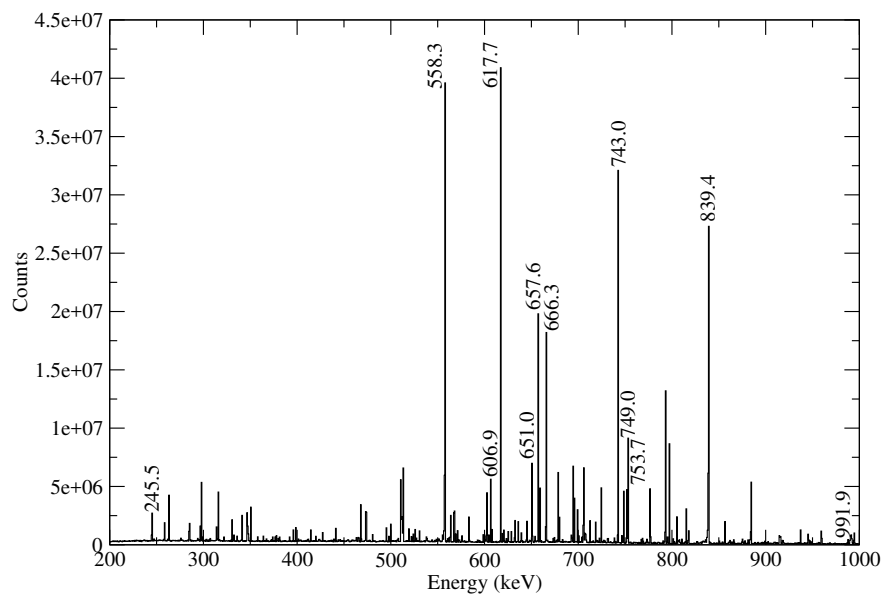

Fig. 6. The truncated $\gamma$-ray spectrum produced in Geant 4 from a ${ }^{252} \mathrm{Cf}$ neutron source and a $1.5 \mathrm{~cm}$ thick CdZnTe detector.

\section{CONCLUSION}

MCNP6 is useful for modelling what reactions are induced by the neutrons, and produces reasonable results for the neutron capture and neutron fluxes. Future work in MCNP may model which other neutron-induced reactions occur in such an experiment. The modelling of secondary radiation is possible with Geant4, and a spectrum which is in line with the experimental data has been produced. In future, the modelling of the electrons produced by the secondary $\gamma$-rays, in a realistic electric field, in possible. In addition, a comparison between these theoretical results and experiment may be undertaken, using the ${ }^{252} \mathrm{Cf}$ neutron source at Lancaster University.

\section{ACKNOWLEDGMENT}

This work is supported by the Engineering and Physical Sciences Research Council (EPSRC), UK, via the Impact Acceleration Account (IAA), in a collaboration between Lancaster University and Innovative Physics Ltd. The contributions of the Lancaster University technical staff are gratefully acknowledged.

\section{REFERENCES}

[1] T. Goorley, MCNP6.1.1-Beta Release Notes, LA-UR-14-24680, 2014.

[2] S. Agostinelli, J. Allison, K. Amako, J. Apostolakis, H. Araujo, P. Arce M. Asai, D. Axen, S. Banerjee, G. Barrand, F. Behner, L. Bellagamba, J. Boudreau, L. Broglia, A. Brunengo, H. Burkhardt, S. Chauvie, J. Chuma, R. Chytracek, G. Cooperman, G. Cosmo, P. Degtyarenko, A. Dell'Acqua G. Depaola, D. Dietrich, R. Enami, A. Feliciello, C. Ferguson, H Fesefeldt, G. Folger, F. Foppiano, A. Forti, S. Garelli, S. Giani, R. Giannitrapani, D. Gibin, J.J. Gmez Cadenas, I. Gonzlez, G. Gracia Abril, G. Greeniaus, W. Greiner, V. Grichine, A. Grossheim, S. Guatelli, P. Gumplinger, R. Hamatsu, K. Hashimoto, H. Hasui, A. Heikkinen, A Howard, V. Ivanchenko, A. Johnson, F.W. Jones, J. Kallenbach, N. Kanaya, M. Kawabata, Y. Kawabata, M. Kawaguti, S. Kelner, P. Kent, A. Kimura, T. Kodama, R. Kokoulin, M. Kossov, H. Kurashige, E. Lamanna, T. Lampn, V. Lara, V. Lefebure, F. Lei, M. Liendl, W. Lockman, F. Longo, S. Magni, M. Maire, E. Medernach, K. Minamimoto, P. Mora de Freitas, Y. Morita, K. Murakami, M. Nagamatu, R. Nartallo, P. Nieminen, T. Nishimura, K. Ohtsubo, M. Okamura, S. O'Neale, Y. Oohata, K. Paech, J. Perl, A. Pfeiffer, M.G. Pia, F. Ranjard, A. Rybin, S. Sadilov, E. Di Salvo, G. Santin, T. Sasaki, N. Savvas, Y. Sawada, S. Scherer, S. Sei, V.
Sirotenko, D. Smith, N. Starkov, H. Stoecker, J. Sulkimo, M. Takahata, S. Tanaka, E. Tcherniaev, E. Safai Tehrani, M. Tropeano, P. Truscott, H. Uno, L. Urban, P. Urban, M. Verderi, A. Walkden, W. Wander, H. Weber, J.P. Wellisch, T. Wenaus, D.C. Williams, D. Wright, T. Yamada, H. Yoshida, and D. Zschiesche, Geant4 - a simulation toolkit, Nucl. Instru. Meth. A 506, 250-303, 2003.

[3] L. Maigne, Y. Perrot, D. R. Schaart, D. Donnarieix, and V. Breton, Comparison of GATE/GEANT4 with EGSnrc and MCNP for electron dose calculations at energies between $15 \mathrm{keV}$ and $20 \mathrm{MeV}$, Phys. Med. Biol., 56, 811-827, 2011.

[4] A.A. Hecht, R.E. Blakeley, W.J. Martin, and E. Leonard, Comparison of Geant4 and MCNP6 for use in delayed fission radiation simulation, Ann. Nucl. Energy, 69, 134-138, 2014.

[5] H. Yang, X. Yang, S. Xiao, N. Satvat and T. Jevremovic, Detection of hidden materials using Nuclear Resonance Fluorescence technique: Simulation and measurements, IEEE Nuclear Science Symposuim \& Medical Imaging Conference, Knoxville, TN, 2010, pp. 531-533.

[6] T.E. Schlesinger, J.E. Toney, H. Yoon, E.Y. Lee, B.A. Brunett, L. Franks, and R.B. James, Cadmium zinc telluride and its use as a nuclear radiation detector material, Mater. Sci. Eng. R., 32, 103-189, 2001.

[7] A. Martín-Martín, M. P. Iñiguez, P. N. Luke, R. Barquero, A. Lorente, J. Morchón, E. Gallego, G. Quincoces, J. M. Martí-Climent, Evaluation of CdZnTe as neutron detector around medical accelerators. Radiat. Prot. Dosimetry, 133, 193-199, 2009.

[8] M. Streicher, S. Brown, Y. Zhu, D. Goodman and Z. He, "Special Nuclear Material Characterization Using Digital 3-D Position Sensitive CdZnTe Detectors and High Purity Germanium Spectrometers," in IEEE Transactions on Nuclear Science, vol. 63, no. 5, pp. 2649-2656, Oct. 2016.

[9] D.S. McGregor, J.T. Lindsay, R.W. Olsen, Thermal neutron detection with cadmium1x zincx telluride semiconductor detectors, Nuclear Instruments and Methods in Physics Research Section A: Accelerators, Spectrometers, Detectors and Associated Equipment, 381, 498-501, 1996.

[10] S. T. Brown, Time-Encoded Thermal Neutron Imaging Using LargeVolume Pixelated CdZnTe Detectors, PhD Thesis, University of Michigan, USA, 2017.

[11] S.F. Mughabghab, Atlas of Neutron Resonances: Resonance Parameters and Thermal Cross Sections. $Z=1-100$, Elsevier, 2006.

[12] K. A. Nelson, N. Edwards, M. J. Harrison, A. Kargar, W. J. McNeil, R. A. Rojeski, and D. S. McGregor, Investigation of CdZnTe and $\mathrm{LiNbO}_{3}$ as electro-optic neutron detectors, Nucl. Instru. Meth. A 620, 363-367, 2010.

[13] J. Blachot, Nuclear Data Sheets for A $=114$, Nucl. Data Sheets, 113, 515-714, 2012.

[14] R. C. Reedy, T. H. Prettyman and N. Yamashita, Backgrounds in cadmium zinc telluride (CZT) gamma-ray spectrometers for planetary science, Abstracts for 43rd Lunar \& Planetary Science Conference (Houston, 19-23 Mar. 2012), \#1284.

[15] M. Chu, S. Terterian, and D. Ting, Role of Zinc in CdZnTe Radiation Detectors, IEEE Transactions on Nuclear Science, 51, 2405-2411, Oct. 2004.

[16] A. B. Smith, P. R. Fields, and J. H. Roberts, Spontaneous Fission Neutron Spectrum of $\mathrm{Cf}^{252}$, Physical Review, 108, 411-413, 1957.

[17] D. Gagnon, G. L. Zeng, J. M. Links, J. J. Griesmer, and F. C. Valentino, Design considerations for a new solid-state gamma-camera: SOLSTICE, IEEE Nuclear Science Symposium and Medical Imaging Conference (Vol. 2, pp. 1156-1160), 2001.

[18] K. B. Parnham, J. Grosholz, R. K. Davis, S. Vydrin, and C. A. Cupec, Development of a CdZnTe-based small field-of-view gamma camera. Proc. SPIE 4508, Penetrating Radiation Systems and Applications III, 134, 2001.

[19] M. Prokesch, D. S. Bale and C. Szeles, Fast High-Flux Response of CdZnTe X-Ray Detectors by Optical Manipulation of Deep Level Defect Occupations, IEEE Transactions on Nuclear Science, 57, 2397-2399, Aug. 2010.

[20] T. Goorley, M. James, T. Booth, F. Brown, J. Bull, L.J. Cox, J. Durkee, J. Elson, M. Fensin, R.A. Forster, J. Hendricks, H.G. Hughes, R. Johns, B Kiedrowski, R. Martz, S. Mashnik, G. McKinney, D. Pelowitz, R. Prael, J. Sweezy, L. Waters, T. Wilcox, and T. Zukaitis, Initial MCNP6 Release Overview, LA-UR 13-22934, 2013.

[21] V. Zerkin, Evaluated Nuclear Data File (ENDF) Database, Internet: https://www-nds.iaea.org/exfor/endf.htm, Mar. 10th, 2017 [May 23rd, 2017]. 
[22] M.B. Chadwick, M. Herman, P. Obložinský, M.E. Dunn, Y. Danon, A.C. Kahler, D.L. Smith, B. Pritychenko, G. Arbanas, R. Arcilla, R. Brewer, D.A. Brown, R. Capote, A.D. Carlson, Y.S. Cho, H. Derrien, K. Guber, G.M. Hale, S. Hoblit, S. Holloway, T.D. Johnson, T. Kawano, B.C. Kiedrowski, H. Kim, S. Kunieda, N.M. Larson, L. Leal, J.P. Lestone, R.C. Little, E.A. McCutchan, R.E. MacFarlane, M. MacInnes, C.M. Mattoon, R.D. McKnight, S.F. Mughabghab, G.P.A. Nobre, G. Palmiotti, A. Palumbo, M.T. Pigni, V.G. Pronyaev, R.O. Sayer, A.A. Sonzogni, N.C. Summers, P. Talou, I.J. Thompson, A. Trkov, R.L. Vogt, S.C. van der Marck, A. Wallner, M.C. White, D. Wiarda, P.G. Young, ENDF/B-VII.1 Nuclear Data for Science and Technology: Cross Sections, Covariances, Fission Product Yields and Decay Data, Nuclear Data Sheets, 112, 2011, 2887-2996.

[23] M.B. Chadwick, M. Herman, P. Obloinsk, M.E. Dunn, Y. Danon, A.C Kahler, D.L. Smith, B. Pritychenko, G. Arbanas, R. Arcilla, R. Brewer D.A. Brown, R. Capote, A.D. Carlson, Y.S. Cho, H. Derrien, K. Guber, G.M. Hale, S. Hoblit, S. Holloway, T.D. Johnson, T. Kawano, B.C. Kiedrowski, H. Kim, S. Kunieda, N.M. Larson, L. Leal, J.P. Lestone, R.C. Little, E.A. McCutchan, R.E. MacFarlane, M. MacInnes, C.M Mattoon, R.D. McKnight, S.F. Mughabghab, G.P.A. Nobre, G. Palmiotti, A. Palumbo, M.T. Pigni, V.G. Pronyaev, R.O. Sayer, A.A. Sonzogni, N.C. Summers, P. Talou, I.J. Thompson, A. Trkov, R.L. Vogt, S.C. van der Marck, A. Wallner, M.C. White, D. Wiarda, and P.G. Young, ENDF/B-VII.1 Nuclear Data for Science and Technology: Cross Sections, Covariances, Fission Product Yields and Decay Data, Nuclear Data Sheets, 112, 2887-2996, 2011.

[24] J. L. Conlin (ed.) and Nuclear Data Team XCP-5, Listing of Available ACE Data Tables, LA-UR-17-20709, 2017.

[25] K. Shibata, T. Kawano, T. Nakagawa, O. Iwamoto, J. Katakura, T. Fukahori, S. Chiba, A. Hasegawa, T. Murata, H. Matsunobu, T. Ohsawa, Y. Nakajima, T. Yoshida, A. Zukeran, M. Kawai, M. Baba, M. Ishikawa, T. Asami, T. Watanabe, Y. Watanabe, M. Igashira, N. Yamamuro, H. Kitazawa, N. Yamano and H. Takano, Japanese Evaluated Nuclear Data Library Version 3 Revision-3: JENDL-3.3, Journal of Nuclear Science and Technology, 39, 1125-1136, 2002.

[26] A. V. Ivantchenko, V. N. Ivanchenko, J-M. Quesada Molina and S. L. Incerti, Geant4 hadronic physics for space radiation environment, Int. J. Radiat. Biol., 88, 171-175, 2012.

[27] J. Blachot, Nuclear Data Sheet for $A=111$, Nuclear Data Sheets 110, 1239-1407, 2009

[28] J. Blachot, Nuclear Data Sheet for $A=114$, Nuclear Data Sheets 113, 515-714, 2012.

[29] S. Lalkovski and F. G. Kondev, Nuclear Data Sheet for A=112, Nuclear Data Sheets 124, 157-412, 2015.

[30] G. Gũrdal and F. G. Kondev, Nuclear Data Sheet for $A=110$, Nuclear Data Sheets 113, 1315-1561, 2012.

[31] J. Katakura and K. Kitao, Nuclear Data Sheet for $A=126$, Nuclear Data Sheets 97, 765-926, 2002

[32] Z. Elekes and J. Timar, Nuclear Data Sheet for $A=1328$, Nuclear Data Sheets 129, 191-436, 2015.

[33] B. Singh, Nuclear Data Sheet for A=130, Nuclear Data Sheets 93, 33242, 2001.

[34] B. Singh, Nuclear Data Sheet for A=64, Nuclear Data Sheets 108, 197 364, 2007. 